\title{
Mixing induced by swift heavy ion irradiation at $\mathrm{Fe} / \mathrm{Si}$ interface
}

\author{
VEENU SISODIA and I P JAIN* \\ Materials Science Laboratory, Centre for Non Conventional Energy Resources, University of Rajasthan, \\ Jaipur 302 004, India
}

MS received 29 January 2004

\begin{abstract}
The present work deals with the mixing of metal and silicon by swift heavy ions in high-energy range. Threshold value for the defect creation in metal Fe calculated was found to be $\sim 40 \mathrm{keV} / \mathrm{nm}$. A thin film of $\mathrm{Fe}(10 \mathrm{~nm})$ was deposited on $\mathrm{Si}(100)$ at a pressure of $4 \times 10^{-8}$ Torr and was irradiated with $95 \mathrm{MeV} \mathrm{Au}$ ions. Irradiation was done at RT, to a dose of $10^{13}$ ions $/ \mathrm{cm}^{2}$ and 1 pna current. The electronic energy loss was found to be $29.23 \mathrm{keV} / \mathrm{nm}$ for $95 \mathrm{MeV}$ Au ions in Fe using TRIM calculation. Compositional analysis of samples was done by Rutherford backscattering spectroscopy. Reflectivity studies were carried out on the pre-annealed and post-annealed samples to study irradiation effects. Grazing incidence X-ray diffraction was done to study the interface. It was observed that ion beam mixing reactions at RT lead to mixing as a result of high electronic excitations.
\end{abstract}

Keywords. Ion beam mixing; irradiation; swift heavy ion.

\section{Introduction}

The formation of silicides due to the defect created at metal/Si interfaces is of great interest owing to its use in inter-connections and contacts in integrated circuit technology and understanding of defect creation is fundamental for electronic device technology. Energetic ion beams are capable in inducing particle transport in a bombarding medium. This phenomenon, called ion beam mixing, has been exploited in many applications, and a great deal of experimental work has been done to find out the characteristic feature of mixing (Paine and Averback 1985; Kim et al 1998; Cheng 1990). Experiments done with metallic targets and thin metallic layer as well as with metallic bilayers have revealed the complexity of ion beam mixing.

Experimental results show that high electronic excitation can also induce structural modifications in metallic systems similar to those in non-metallic systems. This means that all $S_{\mathrm{e}}$-dependent effects induced in different materials are probably related to some basic energy transfer mechanism between the incident ion and the target atoms. Only a limited number of studies have been done in the highenergy range.

Irradiation with $\mathrm{GeV}$ energy ions has been found to produce intermixing in Fe/Si multilayers (Dufour et al 1993). It was shown by Dunlop et al (1994) and Tombrello (1995) that the trails of damage can also be made in metals by high levels of ion energy loss, $S_{\mathrm{e}}$. Threshold $S_{\mathrm{e}}$ for such columnar damage varies from metal to metal. For example, Se for $\mathrm{Fe}$ is $\sim 5 \mathrm{keV} / \AA$ (Dunlop et al 1994). Swift heavy ion induced intermixing in the metallic systems

*Author for correspondence and underlying mechanism responsible for the interdiffusion process is not yet clear. More detailed studies done on the system suggests that for low $(\mathrm{d} E / \mathrm{d} X)$ values, in addition to damage creation, intermixing occurs mainly via thermal diffusion process.

In the present work, Fe/Si bilayer system is studied under the swift heavy ion irradiation purely and effects on the system are observed as a result of $95 \mathrm{MeV} \mathrm{Au}$ ion irradiation.

Mixing is confirmed by characterization of the irradiated and annealed samples using X-ray reflectivity and RBS. Grazing incidence X-ray diffraction technique was employed to study the irradiation effects at the interface.

\section{Experimental}

\subsection{Sample preparation}

Samples were prepared in high vacuum conditions to get controlled properties. In the present study a single layer of Fe [10 nm] of desired thickness was deposited carefully by e-gun evaporation on $5 \% \mathrm{Hf}$ etched $\mathrm{Si}(100)$ at a chamber pressure of $4 \times 10^{-8}$ Torr. UHV system at the Nuclear Science Centre, New Delhi, was employed for the target preparation. The thickness of layer was controlled using the quartz crystal monitor.

\subsection{Irradiation}

The Fe/Si films were irradiated with $95 \mathrm{MeV} \mathrm{Au}$ ions at RT to a dose of $10^{13}$ ions $/ \mathrm{cm}^{2}$ using 15 UD NSC Pelletron. The electronic energy loss has been calculated as $29.23 \mathrm{keV} /$ $\mathrm{nm}$ for $95 \mathrm{MeV}$ gold ions in $\mathrm{Fe}$ and $12.24 \mathrm{keV} / \mathrm{nm}$ in $\mathrm{Si}$ using TRIM calculation. 


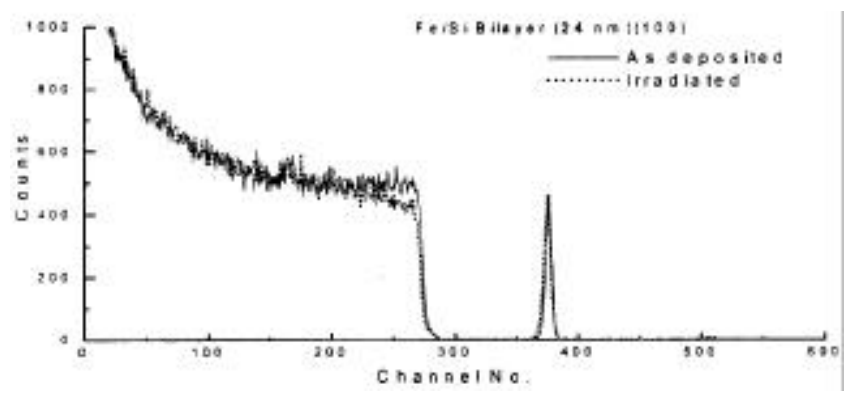

Figure 1. RBS spectra of $\mathrm{Fe} / \mathrm{Si}$ bilayer for as deposited and irradiated specimen.

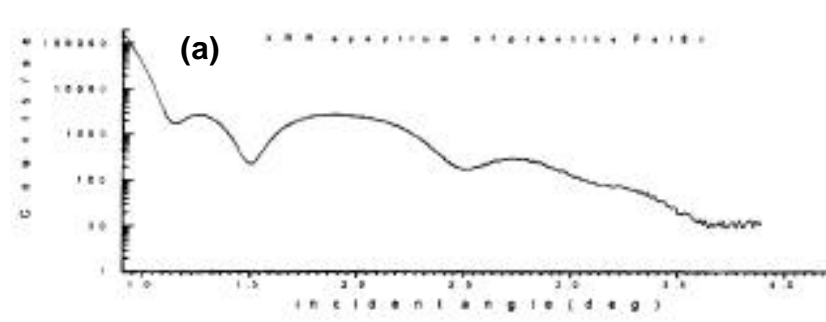

(b)

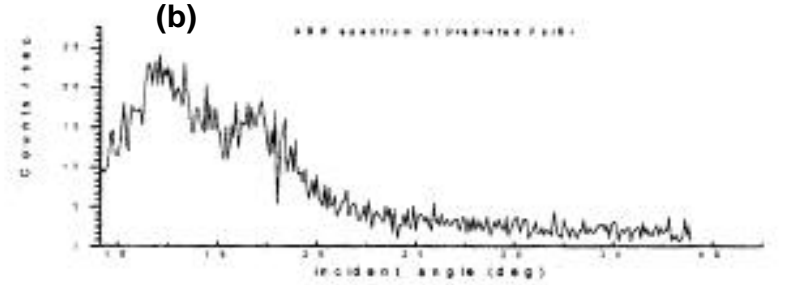

Figure 2. XRR spectra for Fe/Si system: (a) as deposited and (b) after irradiation.

\section{Results}

\subsection{RBS studies}

RBS spectra of Fe thin film over Si (100) for as-deposited and irradiated specimen are shown in figure 1 . The analysis of RBS spectra in both the cases confirms the presence of a thin Fe layer over Si substrate. RBS simulation shows mixing in the Fe/Si system due to swift heavy ion irradiation.

\subsection{XRR studies}

Figure 2 gives the X-ray reflectivity pattern of the $\mathrm{Fe} / \mathrm{Si}$ system in as deposited sample and after irradiation at a dose of $10^{13}$ ions $/ \mathrm{cm}^{2}$. The thickness of the Fe film is confirmed as $\sim 10 \mathrm{~nm}$ from the reflectivity curve of the asdeposited pattern. It is clear from the XRR spectra of irradiated specimen that there is a sufficient damage in the structure due to Au ion irradiation, resulting in mixing.

\subsection{GIXRD studies}

Figure 3 shows the diffraction pattern of the bilayer system. X-ray diffraction pattern of the as-prepared specimen

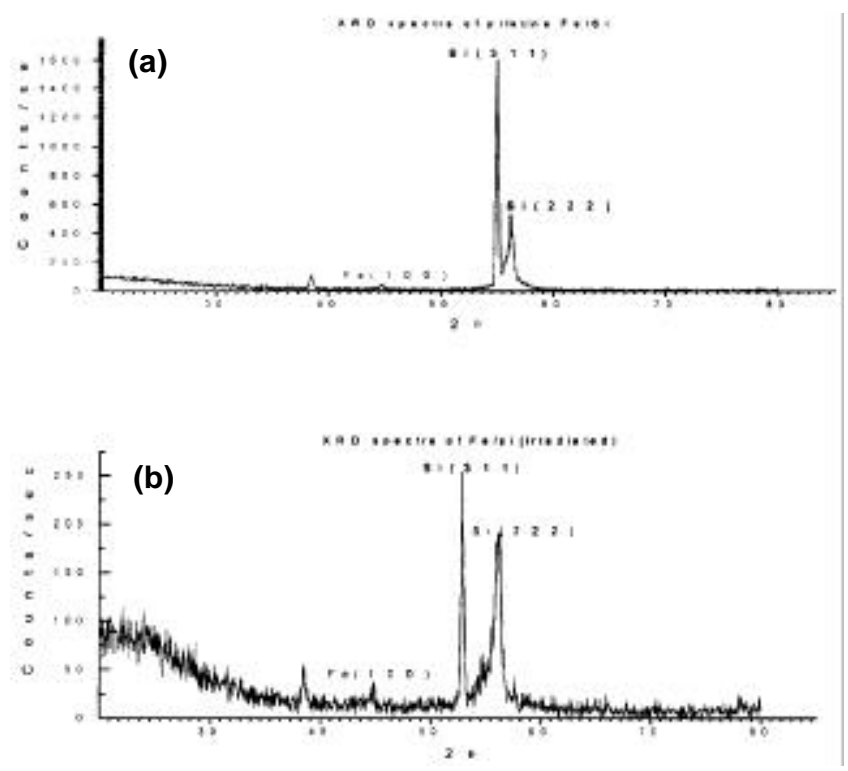

Figure 3. GIXRD spectra of Fe/Si bilayer: (a) as-prepared and (b) for irradiated specimen.

showing Bragg peaks corresponding to metallic Fe (110), $\mathrm{Si}(222)$ and $\mathrm{Si}$ (311) were observed in the $2 \theta$ range of $30-80^{\circ}$. From the GIXRD pattern of irradiated specimen one finds that irradiation by $10^{13}$ ions $/ \mathrm{cm}^{2}$, the height of the crystalline peaks of $\mathrm{Fe}(100)$, reduces by magnitude of the order of $3 \cdot 2$. Also the Bragg peaks corresponding to $\mathrm{Si}$ decreases. This clearly indicates the mixing at the $\mathrm{Fe} / \mathrm{Si}$ interface. The diffraction results indicate the formation of an amorphous phase due to ion beam mixing.

\section{Conclusions}

SHI effects over Fe/Si bi-layer due to irradiation with $95 \mathrm{MeV} \mathrm{Au}$ ions at a low $S_{\mathrm{e}}$ value i.e. below the $S_{\mathrm{e}}$ threshold reported by various studies done in this field, is studied with a dose of $10^{13}$ ions $/ \mathrm{cm}^{2}$. It is found that irradiation in this case results in mixing at the interface. So by taking the advantage of ion beam induced mixing, mixing at interface is considered as a good approach for such systems.

\section{References}

Dufour C, Bauer Ph, Marchal G and Jousset J C 1993 Europhys. Lett. 21671

Dunlop A, Lesueur D and Dammark H 1994 Nucl. Instrum. \& Meth. B90 330

Cheng Y T 1990 Mater. Sci. Rep. 545

Kim S J, Nicolet M A, Averback R S and Peak D 1988 Phys. Rev. B37 38

Paine B M and Averback R S 1985 Nucl. Instrum. \& Meth. B7/8 666

Tombrello T A 1995 Nucl. Instrum. \& Meth. B103 318 